\title{
Technology Capabilities for an Automated and Connected Earthwork Roadmap
}

\begin{abstract}
Purpose:

The development of communication and artificial intelligence technologies has raised interest in connectivity and increased autonomy of automated earthmoving equipment for earthwork. These changes are motivating work to reduce uncertainties, in terms of improving equipment object detection capability and reducing strikes and accidents on site. The purpose of this study is to: (1) illustrates industrial drivers for automated earthwork systems, (2) identifies the specific capabilities which make the transformation happen, and finally (3) determines use cases that create value for the system. These three objectives act as components of a technology roadmap for automated and connected earthwork and can guide development of new products and services.
\end{abstract}

\section{Design/methodology/approach:}

This paper utilized a text mining approach in which the required data was captured through a structured literature review, and then expert knowledge was used for verification of the results.

Findings:

Automated and connected earthwork can enhance construction site, and its embraced infrastructure, resilience by avoiding human faults during operations. Automating the monitoring process can lead to reliable anticipation of problems, and facilitate real time responses to unexpected situation via connectedness capabilities. Research findings are presented in three sections: 1) industrial perspectives, trends, and drivers for automated and connected earthwork, 2) capabilities which are met by technologies, and 3) use cases to demonstrate different capabilities.

\section{Originality/value:}

This study combines the results of disintegrated and fragmented research in the area of automated and connected earthwork and categorises them under new capability levels. The identified capabilities are classified in three main categories including: (1) reliable environmental perception, (2) single equipment decision making toward safe outcomes, and 3) fleet level safety enhancement. Finally, four different LOA are proposed for earthwork technology roadmap.

Index Terms - Drivers, Earthwork, Resilience, Technology innovations, Technology roadmap, Use cases.

\section{INTRODUCTION}

In a globally competitive business environment, technological innovation has significant effects on firms' shortterm performance and long-term viability as technology choices are crucial to competitive advantages (Zhang et al., 2016). When there is uncertainty about the future direction of technology, appropriate technology strategies should be developed to support a firm's future technology expansion plans (Lee, Phaal and Lee, 2013). Emerging supply chains of different technological innovations need strategic and intelligent planning, as innovation development is non-linear, and needs alignment among users (demand) and producers (supply) (Huang et al., 2018). Both perspectives (market and supply chain) are techno-centric since they choose a technology solution initially and then develop prospective pathways, but they are motivated to do this by different drivers. The market perspective is driven by technology adoption using an embedding perspective, whilst the supply chain perspective is motivated to convey a system for individual human needs (Huang et al., 2018). In the construction industry, the primary goal of technology innovation development and automating earthmoving equipment operation has been better performance in terms of higher system safety and higher productivity (Vähä et al., 2013). In other words, strategies should be aligned with industrial agreement on transformation toward autonomy and connectivity in order to motivate technology investment for safe, productive, and resilient construction sites, and earthmoving operation.

In fact, the process of technological innovation takes place through highly complex socio-techno-economic systems, in which regulation and marketing play an important role (Huang et al., 2018). "Technology Delivery System" (TDS) is a technocentric approach for finding innovative content that translates ideas into reality. The technical perspective of TDS points out that $R \& D$ processes are crucial to the development of a new technology, and the key step in this regard is technology roadmapping (TRM) (Huang et al., 2018). Therefore, the strategic alignment can be achieved through developing appropriate technology roadmaps. A technology roadmap has time-oriented associations, and the dependencies over time are bounded by technologies and products. These dependencies extend through relationships with the market, and to responsible organizations for transfer of technologies and products (Geum et al., 2015). In fact, technology roadmaps enable two major ambitions: first it is a planning tool for strategic decision making, second it facilitates communication (Rinne, 2004). Not only that, a technology roadmap is both a planning and forecasting tool, which promotes the integration of the two (Rinne, 2004). A technology roadmap enables organizations to integrate the commercial and technological aspects into the emergence of new technologies (Phaal, 2004).

A technology roadmap represents both management theory and practice (Lee, Song and Park, 2015) and is beneficial at three levels: 1) national research and development: policies that guide technology, science, economics, and innovation 
prospects; 2) industries and sectors: boosting collaboration and coordination in special technological areas; 3) specific technological routes (Zhang et al., 2016). However, technology roadmaps must be capable of continuous enhancement and iterative adaption to accommodate both a short-term and longterm range of views and strategies (Hansen et al., 2016) since they become out of date quickly because of new product developments and disruptive technologies (Lee, Song and Park, 2015). The recent focus in intelligent transportation systems, notably in government-associated research and development activities, has been concentrated on automated vehicle (AV) and connected vehicle (CV) technology roadmaps (Gillespie, 2017; Shladover, 2017) and the divergence in their formation time frames (Shladover, 2017). CV systems have the capability to accept a broad number of information technology system applications, and to closely connect vehicles and infrastructure components into a reasonably-integrated cooperative transportation system. Automated vehicle (AV) systems have had an older unstable history, driven by technology push. AV systems enhance transportation system operations much better when joined with CV systems (Shladover, 2017). These developments have raised the construction industry attention to the autonomy and connectedness of earthmoving equipment such as excavator, but currently there is not a fully developed technology roadmap available for automated and connected earthwork and excavation systems. Past low technology readiness level may have been to blame but this has recently been increased significantly.

Whilst industrial and academic efforts have been made to develop a Connected and Autonomous Plant (CAP) technology roadmap (i3P, Highways England, TRL, 2020), this is broader in scope than excavation and there is no transparent route to advocate CAP utilisation (Highways England, TRL, 2020). Furthermore, technology capabilities have not been very well established or even fully recognized, and the industry faces a huge risk in investing in new systems (Highways England, TRL, 2020). Accordingly, a well-defined roadmap is required for automated and connected earthwork systems which (1) illustrates industrial, social, strategical drivers for associated supply chain and embedded markets, (2) determines scenarios, products, and services that create value for the system, and finally (3) identifies the specific capabilities and resources which make this transformation happen. Establishing technology capability levels will clarify the potential activities that can be handled automatically. For example, utilising accurate and automated monitoring systems provide real-time site awareness capability for excavation system which can lead to accident anticipation, prevention on site and ultimately zero recovery needs. Accordingly, a resilience excavation against accident can be achieved where resilience is defined as "the intrinsic ability of a system to adjust its functionality in the presence of a disturbance and unpredicted changes" (Hosseini, Barker and Ramirez-Marquez, 2016) (p.48). Accordingly, this study aims to (1) illustrates industrial drivers for transition toward automated earthwork systems with a focus on excavation, (2) identifies the specific capabilities which make the transformation happen, and finally (3) determines use cases that create value for the system. These three objectives act as components of a technology roadmap for automated and connected earthwork that contains excavation and can guide development of new products and services.

In the followings, section II explains methods for developing technology roadmaps., as well as the applied approach by the authors' for conducting this research. Section III illustrates the literature findings in three subsections; A) industrial perspectives, trends, and drivers, B) required capabilities, and C) use cases, for automated and connected earthwork systems, with a main focus on excavation as a dominant task. Section IV discusses (1) a new driver for industrial transformation, (2) different categories of technology innovation's capabilities, and (3) different levels of automation for earthwork systems. And finally practical implications and further research requirements are discussed in the conclusion section.

\section{METHOD}

This section explains the standard methods for developing technology roadmaps, and introduces the authors' approach for collecting and synthesizing data to achieve the above mentioned objectives.

\section{A. Methods for developing technology roadmaps}

A literature search strategy was created and executed in order to comprehensively understand the concept of "technology roadmap", and to identify the scientific methods for developing a technology roadmap. This search strategy is illustrated in Table I, and it was applied in Google Scholar and Science Direct. The following insights about conceptualizing technology roadmap as well as associated methods was extracted from articles found by this search strategy.

$$
\text { Table I }
$$

Search strategy used to understand roadmap concept and to identify associated methods.

\begin{tabular}{lll}
\hline \hline String & Keywords & $\begin{array}{l}\text { Boolean } \\
\text { Operator }\end{array}$ \\
\hline 1 & (automation OR technology) & AND \\
2 & "roadmap" & AND \\
3 & (trend* OR pathway* OR feature*) & \\
\hline \hline
\end{tabular}

Various methods can be used to capture the information needed for the technology roadmap, including: qualitative, quantitative and hybrid methods. Qualitative methods include: expert interview, Delphi, discussions, seminars/workshops, often connecting academic researchers, industrial stakeholders, and government officials. Quantitative methods include: text mining, bibliometric, computer-based graphical techniques, artificial intelligence, intelligent information techniques, pattern recognition, and machine learning. Qualitative methods have not adequately utilized statistical models for computation as they rely more on expert knowledge, whilst quantitative methods are intelligent techniques to generate automatic mapping for text mining, since expert knowledge does not play a significant role in it. A hybrid method benefits from a 
combination of both. Technology roadmap that take significant account of expert knowledge (Geum et al., 2015), provide credible responsibility for the outcomes. On the other hand, quantitative methods are able to balance the possible expert favouritism (Zhang et al., 2016).

Patent analysis is an alternative method that raises awareness of technological development trends (Kim et al., 2009; Lee, Phaal and Lee, 2013). It is important to notice that along with academic papers review, patent analysis provides a higher cognizance of technological development trends (Lee, Phaal and Lee, 2013)(Gausemeier, Fink and Schlake, 1998). For example, Kim et al (Kim et al., 2009) conducted patent analysis in the construction industry that relied on keywords established by expert opinion and literatures, and a patent map was produced based on these keywords.

For collecting and analysing data, it is important to know that a technology roadmap has three main layers: market, product and technology. The interaction and relationships of these layers requires precise analysis (Geum et al., 2015)(Gausemeier, Fink and Schlake, 1998)(Lee and Geum, 2017). Due to the different characteristics of the technology roadmap layers, it is not sufficient to develop a technology roadmap using a single method (Lee and Geum, 2017). Market planning analysis and evaluation requires external scenarios that are uncontrollable, while internal scenarios are primarily strategic, controllable product and technology planning decisions. In addition, more recently, researchers have concentrated on the application of roadmaps on the paradigm of service, which means adding a new layer to the existing technology roadmap in terms of "Services", which has the same standing as the product layer (Lee, Phaal and Lee, 2013). In fact, service science constructs a conceptual base for serviceoriented business models to build a robust and flexible IT-based business models that respond efficiently to the needs of distinct customers. Accordingly, servitisation refers to the ability to improve the organization's capability to acquire more valuable products by integrating planning processes, providing services and production value (Lee, Phaal and Lee, 2013).

In addition, a robust and reliable evolutionary planning of technology and market should take account of uncertainties embedded in socio-technical-economic systems. Scenario planning is a tool currently used to deal with uncertainty which attempts to anticipate hypothetical futures (Gausemeier, Fink and Schlake, 1998). Technology foresight has been implemented by both approaches: technology roadmap, and scenario planning. These approaches should be integrated to deal best with the dynamic and uncertain nature of the business environment (Lee and Geum, 2017) (Hussain, Tapinos and Knight, 2017).

In fact, technology roadmaps consider a particular scenario which cannot be certain over a period of time, due to unpredictable and emergent changes in environments and markets (Hussain, Tapinos and Knight, 2017). In contrast, in scenario-based roadmaps, future foresight is derived by exploring the broad environment and issues that influence technology (Hussain, Tapinos and Knight, 2017). In other words, there is a high level of uncertainty in developing new technologies since future conditions of complex environments are unpredictable (Hansen et al., 2016). This put markets in risk thus appropriate responses to future conditions are required to avoid difficulties or failures of technology markets and investors (Hansen et al., 2016). Accordingly, scenario planning should be integrated into technology road mapping to be prepared for uncertain futures.

Nevertheless, various studies have been trying to develop technology roadmaps for different industries that aim to adapt available technology innovations efficiently and in a timely way in order to maintain competitiveness. Each study considered a specific aspect or method, or a specific case study. For example, Hansen et al., (2016) identified the market drivers, new systems, and technologies of rail automation through literature review and verified them by expert judgments. Lee, Phaal and Lee, (2013) showed how to use the mapping processes to explore the potential convergence of future services and products in a smart city development case. Zhang et al., (2016) proposed the importance of a hybrid roadmaping technique that integrates several approaches into a multi-level approach in order to employ transparent techniques to create best practices for emerging fields.

For the technology roadmap of automated and connected systems, there are numerous studies and popular technology roadmaps for interconnected and automated vehicle systems. As a result, a large ecosystem has been created for connected and automated vehicle (CAV), and several testbeds have been provided to bring the concept of CAV into reality. For example, ZENZIC has shaped the ecosystem for CAV in the UK and established the "UK connected and automated mobility (CAM) roadmap to 2030". This organization unified industry, government and academia that formed the CAM testbed in the UK to set standards in this field. This trend along with availability of advanced technologies have prompted the rise of interconnected and autonomous construction techniques. Accordingly, Connected and Autonomous Plant (CAP) Roadmap to 2035 was developed by Highways England, TRL, and i3P to provide tools to support CAP planning, strategy and investment decisions (Highways England, TRL, 2020). However, there are still challenges and concerns about the functions of the current technologies' capabilities, and there is also a lack of a unified direction of automation (Highways England, TRL, 2020). This paper addresses these challenges through identifying available technology capabilities for automated and connected earthwork roadmaps, which are designed to predict and avoid accidents during operations.

\section{B. Applied method}

This paper utilized a text mining approach in which the required data was captured through a structured method: literature review and then findings verified by expert knowledge in several workshops.

A search strategy was used to specifically identify existing technologies for automated and connected earthwork that include excavation, see Table II. Scopus, PQ/ABI, Web of science, EBSCOhost were searched based on their relevance to engineering and business areas. 49 relevant papers were chosen based on title, and abstract review, and finally 32 papers were chosen for inclusion based on a full text review. The data 
provided by the search results allowed analysis of (a) industrial perspectives, trends, and drivers; (b) capabilities and capability types; (c) possible use cases. Given our search criteria in Table II, in scope are only those technologies which enhance systems safety and resilience, in terms of reducing accidents during excavation; other technologies are outside the scope of this work.

\section{Table II}

Search strategy used to identify technology innovations for excavation operation.

\begin{tabular}{lll}
\hline \hline String & Keywords & $\begin{array}{l}\text { Boolean } \\
\text { Operator }\end{array}$ \\
\hline 1 & $\begin{array}{l}\text { (automat* OR technology* OR automat* OR } \\
\text { intelligent*) } \\
\text { (resilient* OR } \text { OR safety OR "resilience }\end{array}$ & AND \\
2 & $\begin{array}{l}\text { engineering") } \\
\text { (excavate* OR "earthmove* equipment*") }\end{array}$ & \\
3
\end{tabular}

\section{FINDINGS}

This section presents literature review findings in three subsections which are 1) industrial perspectives, trends, and drivers, 2) capabilities which are met by technologies, and methods to detect objects during earthwork and avoid accidents, and 3) use cases to demonstrate different capabilities.

\section{A. Industrial perspectives, trends, and drivers}

The automation notion began in the manufacturing industry and has had a remarkable impact on a broad range of other industries such as mining and construction. The major benefits of construction automation are productivity improvement, quality and reliability, safety, enhancement of working conditions, savings in labour costs, standardization of components, life cycle cost savings, and simplification of the workforce, and etc. (Vähä et al., 2013). The main ones that have been discussed in literature are presented in Table III. Since the significance of anticipated benefits could pan out in construction industry, they act as drivers for technology development and adoption. These benefits were identified as the main drivers for CAP development and adaption (i3P, Highways England, TRL, 2020).

\section{B. Capabilities for safe earthwork}

As mentioned earlier, establishing technology capability levels will clarify the potential activities that can be handled autonomously and safely. Therefore, this section presents and categorises the identified capabilities for safety of automated earthwork during excavation and the associated technologies and methods that were developed to create a safe excavation system.

\section{1) Object detection capabilities to avoid site accidents}

Automated data capturing technologies such as Global Positioning System (GPS), Radio Frequency Identification (RFID), Laser Detection and Ranging (LIDAR), UltraWideband (UWB), and video/audio capturing systems which are called "Real-Time Location Systems" (RTLSs) emerged to overcome deficiencies of traditional monitoring methods on construction sites (Olhoeft, 2000; Chae and Yoshida, 2010;
Vahdatikhaki, Hammad and Siddiqui, 2015; Vahdatikhaki et al., 2017; Awolusi, Marks and Hallowell, 2018; Soltani, Zhu and Hammad, 2018). This is the main area that sensor systems have been used for automating excavation systems.

Real time positioning devices such as GPS and UWB are used as automated vehicle tracking technologies to provide 3D locations of equipment and stationary plant which are a valuable data for planning and management of resources (Soltani, Zhu and Hammad, 2018)(Dou et al., 2016). Many technologies are available that can capture equipment pose data such as laser-based methods, inertial measurement unit (IMU), and active and passive marker-based motion tracking systems (Soltani, Zhu and Hammad, 2017). Location data captured by RTLSs can facilitate the identification of machine-induced safety hazards and the analysis of operation productivity (Vahdatikhaki, Hammad and Siddiqui, 2015). Location-based guidance systems (LGSs) were produced by integration of RTLS geo-positioning data, 3D design models, and digital terrain model (DTM) to support equipment operators. This support can be a cabin-mounted display as a visual guidance to operators, or control of the equipment movement and position (Vahdatikhaki et al., 2017). Automated machine control and guidance $(\mathrm{AMC} / \mathrm{G})$ are accessible LGSs in the market which are adopting expensive high-accuracy GPS. UWB is an alternative cost-effective technology which can be used in RTLS systems (Vahdatikhaki et al., 2017).

Investment into technologies with capabilities for detection and recognition of all kind of construction resources has an important burden in achieving fully automated excavation (Tajeen and Zhu, 2014). Computer-vision based methods has been significantly used to automatically monitor and detect people, buildings, plant, materials and equipment on excavation sites from images or videos to improve safety and productivity (Fang et al., 2018; H. Kim et al., 2018). This is the area that machine learning needs to be applied to advance the accuracy of object detections. Accordingly, high-resolution digital wireless cameras and high capacity storage devices have been adopted to a great extent at construction sites as a result of their good enough return on investment, as well as producing helpful management information for construction engineers/managers to monitor and control sites remotely and dynamically (Tajeen and Zhu, 2014).

In addition, wearable sensing devices such as physiological sensors are another kind of technology which can greatly improve occupational safety and health in hazardous construction areas which requires human physical intervention such as excavation sites (Awolusi, Marks and Hallowell, 2018). A smart vest with an embedded indoor GPS and a wristband with physiological sensors are two types of wearable devices for location and activity tracking. GPS, accelerometer or IMU can broaden the technology application by showing human movements while tracking his/her location.

2) Object detection capabilities to avoid below the ground utility strikes

An excavator strike of a buried utility network or other resource results in damage to pipes and cables, worker injuries and deaths. Various geophysical sensors have been utilized to 
Table III

Main Drivers for autonomous and Connected Excavation.

\begin{tabular}{|c|c|c|}
\hline & Drivers & Reference \\
\hline 1 & Productivity & $\begin{array}{l}\text { (Vähä et al., 2013)(Vahdatikhaki et al., } \\
\text { 2017)(Awolusi, Marks and Hallowell, } \\
\text { 2018)(Soltani, Zhu and Hammad, } \\
\text { 2018)(Soltani, Zhu and Hammad, } \\
\text { 2017)(Tajeen and Zhu, 2014)(Fang et } \\
\text { al., 2018)(H. Kim et al., 2018)(Su et } \\
\text { al., 2013)(Azar and Kamat, } \\
\text { 2017)(Zekavat, Moon and Bernold, } \\
\text { 2014)(J. Kim et al., 2018)(Zhang and } \\
\text { Hammad, 2011) (Rezazadeh Azar and } \\
\text { McCabe, 2012) } \\
\text { (Jabri and Zayed, 2017)(Rezazadeh } \\
\text { Azar and McCabe, 2011)(Hammad } \text { et } \\
\text { al., 2012) }\end{array}$ \\
\hline
\end{tabular}

$2 \quad$ Safety

$3 \quad$ Quality

4

Standardization

Life cycle cost savings
(Vähä et al., 2013)(Shladover, 2017) (Vahdatikhaki, Hammad and Siddiqui, 2015)(Vahdatikhaki et al., 2017) (Chae and Yoshida, 2010)(Soltani, Zhu and Hammad, 2018)(Soltani, Zhu and Hammad, 2017)(Fang et al., 2018) (H. Kim et al., 2018)(Zhang et al., 2017)(Talmaki and Kamat, 2014) (Behzadan, Dong and Kamat, 2015)(Su et al., 2013) (Azar and Kamat, 2017)(Zekavat, Moon and Bernold, 2014)(J. Kim et al., 2018) (Choi, Hwang and Lee, 2017) (Zhang and Hammad, 2011) (Rezazadeh Azar and McCabe, 2012)(Jabri and Zayed, 2017)(Li, Cai and Kamat, 2015)(Rezazadeh Azar and McCabe, 2011)(Jo et al., 2017)(Hammad et al., 2012)(Vahdatikhaki and Hammad, 2015)

(Vähä et al., 2013) (Shladover, 2017)(Vahdatikhaki, Hammad and Siddiqui, 2015)(Vahdatikhaki et al., 2017)(Tajeen and Zhu, 2014)(Fang et al., 2018)(H. Kim et al., 2018) (Talmaki and Kamat, 2014) (Behzadan, Dong and Kamat, 2015) (Su et al., 2013)(Viljamaa and Peltomaa, 2014)(Zekavat, Moon and Bernold, 2014)(J. Kim et al., 2018) (Choi, Hwang and Lee, 2017) (Zhang and Hammad, 2011)(Hammad et al., 2012)

(Vähä et al., 2013)(Vahdatikhaki et al., 2017)(Awolusi, Marks and Hallowell, 2018)(Zhang et al., 2017) (Behzadan, Dong and Kamat, 2015)

(Vähä et al., 2013)(Shladover, 2017) (Vahdatikhaki, Hammad and Siddiqui, 2015)(Vahdatikhaki et al., 2017) (Awolusi, Marks and Hallowell, 2018) (Olhoeft, 2000)(Dou et al., 2016) (Tajeen and Zhu, 2014)(Talmaki and Kamat, 2014)(Su et al., 2013)(Azar and Kamat, 2017)(Viljamaa and Peltomaa, 2014)(Li, Cai and Kamat, 2015) (Awolusi, Marks and Hallowell, 2018) locate buried utilities, such as passive magnetic fields for electrical cable detection, vibro-acoustic methods for pipe detection, incorporated small sensors for water pipe detection, low-frequency electromagnetic sensors, and Ground Penetrating Radar (Dou et al., 2016). Despite development of different kind of sensors, detecting utility network under the ground during excavation has been a difficult and challenging task, and further sensor evolution and more advance methods are required in this regard.

\section{3) Digitalization, data fusion and visualization}

The ultimate aim of automated and connected equipment is moving toward an autonomous system which requires comprehensive environmental perception for reliable decision making. Construction sites are a complex and unstructured environment containing various static and dynamic objects. Location and state of all the objects cannot be captured by a single technology/sensor. Therefore, combination of a few sensors are required for reliable environmental perception. Data fusion is a process in which acquired signals from multiple sensors are integrated based on some algorithms to facilitate decision making by improving system reliability, and reducing fuzziness of information (Zhang et al., 2017). Quantitative and qualitative methods have been used for data fusion such as rough set, maximum entropy approach, fuzzy integral, Dempster-Shafer (D-S) evidence theory. For example, in detecting utility network below the ground single geophysical technique is not sufficient to identify all types utility in varying soil conditions (Talmaki and Kamat, 2014). Hence the multisensory approach can be used which is based on combined application of geophysical technologies. If multi-sensor data is integrated appropriately, a more accurate and complete buried utility network representation can be built (Fang et al., 2018) (Kolera and Bernold, 2006).

On the other hand, information technology approaches are required to achieve the full potential benefits of multisensory approach. The information technology approach combines computer graphics visualization, geospatial databases, and tracking technology to depict the position of the subsurface utility lines relative to the equipment operator and the excavation crew (Talmaki and Kamat, 2014). For example, information technologies such as web-based services are using geospatial databases to store buried utility data and display them to the user in two-dimensional (2D) or three-dimensional (3D) visualization on desktop workstations, smart phones, tablets, and personal digital assistants. Or augmented reality (AR) has been used to visualize underground utilities to assist utility inspection and maintenance, as well as improving visual perception for buried utility and excavation safety (Behzadan, Dong and Kamat, 2015). By utilizing AR, the user receives a mixed view of the real world and virtual underground utility lines (Talmaki and Kamat, 2014).

\section{4) Accuracy and uncertainty detection capabilities}

A crucial step toward developing technology innovations for automated and connected excavation is capturing uncertainties associated with the data, models, systems and associated 
approaches. A model's uncertainty can be represented by attributes relating to incompleteness or inaccuracy, probability distributions, missing parameters, etc. (Su et al., 2013). Information and communication technologies such as virtual reality (VR) and AR can facilitate uncertainty visualization (Behzadan, Dong and Kamat, 2015).

5) Data and connectedness related capabilities

An important condition approaching autonomous operation of excavators is machine-to-machine (M2M) communication platforms and data sharing of earthmoving fleet (Azar and Kamat, 2017). There are multiple barriers for M2M communication, including real-time intake of data, database maintenance, cyber security, privacy and proprietary data issues. Big data analysis technologies are needed to provide a large amount of data, and machine learning techniques can facilitate pattern recognition and decision making. It is essential to develop systems with ability to deal with diverse data sources, and define mechanism to create complex models that integrate design and as-built data based on leading communication technologies and machine control utilization (Viljamaa and Peltomaa, 2014). Advanced wireless communication technologies have facilitated automatic near real-time data exchange (Zekavat, Moon and Bernold, 2014)(Viljamaa and Peltomaa, 2014). For autonomous operation of an excavator, wireless communication system should be able to handle earthmoving's site long range communication and equipment cooperation while numerous sensors are utilized for the system operation, and the acquired data from sensors needs to be transferred to different modules (J. Kim et al., 2018).

\section{6) Multi-agent coordination}

Technology innovations and coordination strategies are required to enhance fleet level safety during excavation, rather than just focusing on technology development for a single equipment (Vahdatikhaki et al., 2017). This has higher importance when human interventions in the process are required. Recently the combination of human-in-the-loop cyber-physical systems (HiLCPSs), with building information modelling (BIM), makes it possible to gain proactive improvements of operational safety by setting up activity-level operation planning, and management (Zhang et al., 2017) (Choi, Hwang and Lee, 2017). HiLCPSs facilitates human intervention in the process through reciprocal communication by providing a human-machine interface (Jirgl, Bradac and Fiedler, 2019). These developments facilitate execution of different multi-agent coordination approaches in real-time. The decision making process and information exchange can happen through three different multi-agent coordination approaches including: 1) communication within a centralized architecture; 2) communication within a distributed (decentralized) architecture; and 3) communication within a hybrid architecture which is a combination of centralized and distributed forms (Zhang and Hammad, 2011).

\section{Use cases-practical implications}

A number of use cases are identified based on literature review and expert knowledge. These define functionalities for excavation to meet expectations of autonomous operation and demonstrate the desired values and services (financial, social, and environmental) to address the challenges for achieving safe and resilient excavation system. Scenarios which are used for technology roadmaps may represent alternative use cases. Use cases describe a sequence of events to accomplish a goal. The goal of a use case is met by excavation capabilities discussed in section IIIB. The goals in no particular order are: real time pose and state identification; automated actual productivity calculation; utility network data visualization; real time dynamic mapping; accident prediction and prevention; collision free motion and path planning; and real time decision making. The tabulation in Table IV demonstrates that all capabilities from section IIIB are essential for all use cases, except that multi-agent coordination is required only for the real time decision making use case.

Table IV

Preconditions for use cases.

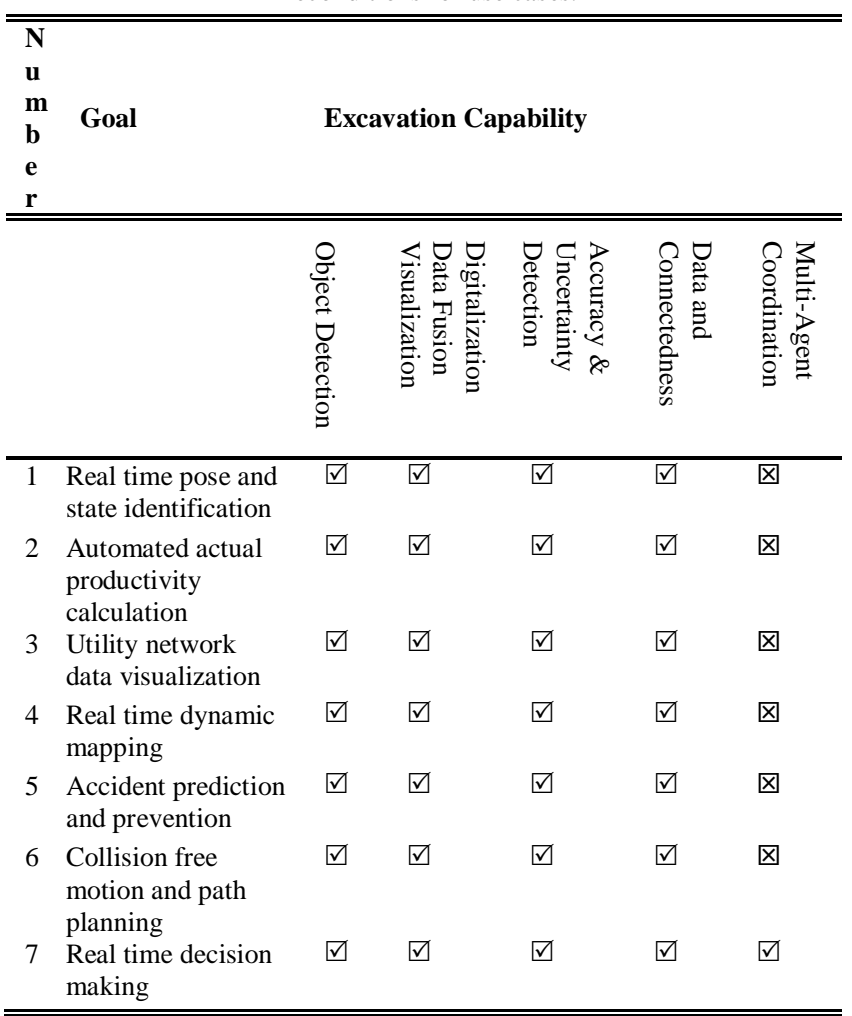

The seven use cases are detailed in Table V (a) to (g). Each use case has a name which reflects the purpose or goal being achieved by the use case and which is described more fully under 'Application'. The normal sequence of events is described under 'Basic Flow'.

\section{Discussion}

Automated earthwork's technology roadmapping promotes an evolutionary planning of technology and offers technology and earthmoving equipment's markets coevolution (Rinne, 2004). As the scale and scope of technology roadmaps extend, so does the need for collaboration, alternatively roadmap integration facilitates and broadens the context of innovation by 
bringing components together from various roadmaps (Rinne, 2004). This paper contributes in developing the CAP furthermore by providing the following components:

Table $V(a)$

Use Case 1

\begin{tabular}{ll}
\hline \hline Goal/name & REAL TIME POSE AND STATE IDENTIFICATION
\end{tabular}

Application Knowing the near real-time pose of the earthmoving equipment is an important necessity for improving safety and productivity of excavation operation. A safer excavation system can be achieved by accurately capturing the movements of the equipment as well as their individual parts using motion tracking techniques (Soltani, Zhu and Hammad, 2017).

Basic Flow Excavator is continuously tracking its spatial position and relative orientation which is called equipment pose (Vahdatikhaki et al., 2017)(Azar and McCabe, 2012). The pose of the excavator is also an indicator of its working state (Azar and McCabe, 2012). The excavator pose shows if it is idle or working, and if working it clearly shows the working states such as moving, loading, swinging, and etc. (Azar and Kamat, 2017). In addition, the excavator's individual parts' speed is detectable.

Table $V(b)$

Use Case 2

Goal/name AUTOMATED ACTUAL PRODUCTIVITY CALCULATION

Application The cycle time as well as the equipment pose information helps to calculate the actual time for different state of operations, and ultimately calculate its productivity automatically (Soltani, Zhu and Hammad, 2017)(Azar and Kamat, 2017). This also facilitates automatic future performance anticipation, maintenance planning, and even budget management (Jabri and Zayed, 2017).

Basic Flow Task execution is monitored automatically and is checked to make sure the tactical plan is followed by the equipment accurately, and the cycle time of the equipment is calculated (Vahdatikhaki et al., 2017). Accordingly, the required number of equipment that should be on site concurrently is optimized to avoid unnecessary dense population of equipment that boost accident probability.

Table V(c)

Use Case 3

Goal/name $\quad$ UTILITY NETWORK DATA VISUALIZATION

Application The situation of the excavator related to utilities lines on the spatial map can be estimated to indicate their proximity (Dou et al., 2016).

Basic Flow Utility supplier provides as-built, statutory records to create a static spatial map of the utility network. This static map is deployed as a resource to the excavator operator. GPS technology and technology onboard the excavator (e.g. a programmable tablet) (Talmaki and Kamat, 2014) are also added to indicate the proximity of excavator and utility network.
Table $V(d)$

Use Case 4

\begin{tabular}{ll}
\hline \hline Goal/name & REAL TIME DYNAMIC MAPPING
\end{tabular}

Application The situation of the excavator related to utilities lines on the spatial map can be estimated to indicate their proximity. It is very important to record objects' positions and orientations in real-time for dynamic path planning. Both static and dynamic map contain some level of uncertainty and incompleteness due to quality of the data captured ( $\mathrm{Li}$, Cai and Kamat, 2015). However, static and dynamic map can be integrated to overcome current limitations of each.

Basic Flow Excavator generates a dynamic map of detected objects during its operation. This dynamic map, which have the site topology as its core layer, include various layers based on properties and attributes of different objects. The first layer presents over the ground utilities (such as telegraph poles), the second layer localize dynamic equipment around excavator (H. Kim et al., 2018)(Rezazadeh Azar and McCabe, 2011) (might have different layers for different equipment such as truck, loader, crane, etc.), the third layer is allocated to detected humans, and the fourth layer to localize utility network below the ground (again might form a few layers for different types of utility network below the ground, electricity, gas, water, etc.).

\begin{tabular}{|c|c|}
\hline & $\begin{array}{l}\text { Table V (e) } \\
\text { Use Case } 5\end{array}$ \\
\hline Goal/name & "ACCIDENT PREDICTION AND PREVENTION \\
\hline Application & $\begin{array}{l}\text { Initial design and determination of proximity zone } \\
\text { around an excavator should take account of different } \\
\text { types of objects, their static or dynamic states, and } \\
\text { critical points of contact. Critical points of contact } \\
\text { can be defined as points/parts of an equipment, with } \\
\text { high probability of contact and encountering with } \\
\text { other objects. }\end{array}$ \\
\hline Basic Flow & $\begin{array}{l}\text { Excavator generates a number of dynamic proximity } \\
\text { Zones (Azar and Kamat, 2017)(Jo et al., 2017) } \\
\text { around itself based on its shape and specification. } \\
\text { Different proximity zones and warning alerts are } \\
\text { defined for different objects around the excavator. } \\
\text { An initial large proximity zone is defined based on } \\
\text { the excavator articulated shape, and speed. When the } \\
\text { excavator detects and identifies an object within this } \\
\text { initial proximity zone, another proximity zone } \\
\text { designed specifically for the class of the identified } \\
\text { object is activated. Generally, excavator is able to } \\
\text { create three specific proximity zones including: 1) a } \\
\text { proximity zone to prevent accident with human } \\
\text { which is based on human skeleton and movement } \\
\text { dynamics, 2) a proximity zone to prevent accident } \\
\text { with other equipment which is based on equipment } \\
\text { types, functions, and movement dynamics, and 3) a } \\
\text { proximity zone to prevent utility strike. }\end{array}$ \\
\hline
\end{tabular}


Table $V(f)$

Use Case 6

Goal/name COLLISION FREE MOTION AND PATH PLANNING

According to [(H. Kim et al., 2018), P (3 of 12)] "a path planning algorithm computes a trajectory from the equipment present pose to a desired future pose". An autonomous excavator should be capable of real-time safe motion and path planning in a complex environment with static and dynamic obstacles. For active multi-equipment motion planning and optimized viable path the work space arrangement should be done by continued collision detection inquiries (Hammad et al., 2012)

Basic Flow Autonomous excavator will be capable of re-path planning in an adequate long-enough duration of time rather than just avoiding immediate potential collisions which can lead to stopping equipment and consequently lower productivity (Hammad et al., 2012). This approach which is called Look ahead equipment workspace is capable of indicating the safe portion of the site by considering the future state of other equipment and workers, associated proximity risks, and visibility risks over the next few seconds (Vahdatikhaki and Hammad, 2015).

Table $V(g)$

Use Case 7

Goal/name $\quad$ REAL TIME DECISION MAKING

Application In a higher level of autonomy, the excavator should be able to do the trade-offs between safety, productivity, and resilience in complex construction environment. The complicated decision making which is based on several safety and productivity and resilience parameters, will happen through highly accurate and fast information exchange and communication on construction site.

Basic Flow Excavator is able to communicate with various objects around it in the same time, including above the ground objects and below the ground objects. All-inclusive communication contains excavator to equipment, excavator to human, and excavator to utility network communication (Vahdatikhaki et al., 2017). The excavator receives and sends partial realtime information about critical parameters (Vahdatikhaki et al., 2017) such as spatial position, pose and state, and speed of its different parts from and to the objects entering its proximity zone. The equipment needs to record its performance for further learning from experience. This is an area for artificial intelligence development. It can be seen from the Table III a multi-agent coordination strategy is necessary in this case.

\section{A. Driver for automated earthwork}

The field of construction automation applies the industrial automation principle to automate construction processes. Advanced robots and equipment were invented to perform specific tasks traditionally done by humans. On excavation sites, robots that share work spaces with workers must be set for high level of safety, and have perceptive user interfaces.

Furthermore, a fundamental robot characteristic for excavation environment is flexibility to be able to function in unstructured and dynamic environment conditions of construction site (Vähä et al., 2013).

Accordingly, the main drivers for automated and connected earthwork are the ones illustrated in Table III but the impact of automated and connected earthwork on the system's resilience has been neglected. Construction sites encompass parts of infrastructure systems and operations including transportation systems, utility networks, etc. Such infrastructure systems are governed by legal and regulatory requirements. Most infrastructure regulation is concerned with public health and safety. In fact, governments ensure that public infrastructure are safe systems as their safety leads to social and economic prosperity. However, the idea of a resilient construction site has much more positive impact on an infrastructure system and wider society and economy, and it is a key step toward public health and safety improvement. The impact is important as a resilient system -a resilient excavation system- has the capability to continue operations in the event of expected and unexpected hazards and accidents (Hollnagel, 2014), rather than simply eliminating the excepted hazards in the site.

In fact, conventional safety management aims to design and sustain a condition that the possibility of negative risks such as near misses, and accidents are as small as possible. The conventional safety management perspective believes that systems are well-known and perform well, which is not the case in construction sites. On the other hand, resilience engineering tries to understand a system's performance and all the conditions in general, not only failures and the negatives outcomes (Hollnagel, 2014)(Huber et al., 2007)(Macchi, Hollnagel and Leonhard, 2009). It tries to increase a system's capability to succeed in different conditions. It means a resilient system should be able to respond, to monitor, to learn and to anticipate expected and unexpected conditions(Hollnagel, 2014)(Huber et al., 2007). Earthmoving equipment such as excavators in construction sites need to cope with the complexity of an unstructured and dynamic environment. Unstructured and dynamic environments and systems contain large uncertainties and conventional safety management is not able to deal with uncertainties. Therefore, there is a need to move from conventional safety management toward resilience engineering approach as a novel safety management paradigm (Shirali et al., 2012) which views safety as a dynamic aspect and establishes how a system operates despite disruptions, and it is a substitute method to deal with safety concerns, risks, and accidents (Hollnagel, 2014).

Most accidents are the result of human behaviour such as operator poor decision making abilities, or a late reaction to unanticipated situation. Automated and connected earthwork systems can enhance construction site, and its embraced infrastructure, resilience by avoiding human faults during operations as a result of automating the monitoring process which can lead to reliable anticipation of problems, and facilitate real time responses to unexpected situation via connectedness capabilities, and learn from experience to avoid the same dangerous behaviour in future. These act as important 
incentives and drivers for developing automated and connected earthwork systems.

\section{B. Capability classification}

The capabilities identified for automated and connected earthwork systems in section IIIB are classified in three main categories including: 1) reliable environmental perception, 2) single equipment decision making toward safe outcomes, and 3 ) fleet level safety enhancement. The first category requires monitoring and object detection technologies to avoid accident on site, and to avoid below the ground utility strike during excavation. The second category needs (1) high level of accuracy in monitoring data, (2) visualization techniques, and (3) uncertainty detection methods. The integration of multisensory data approaches and information technologies is a big step toward visualization of accurate data that currently needs further developments. And finally for the third category, digitalization and connectedness related facilities are required along with appropriate multi-agent coordination strategies.

All three categories of capabilities are essential, interrelated, and play an important role in achieving automated and connected earthmoving operations faster. For example, accurate environmental perception facilitates equipment decision making toward safety which is an important parameter for fleet level safety enhancement. In addition, advances in digitalization and connectedness technologies reinforce visualisation of multisensory data. On the other hand, efficient multi-agent coordination approaches help to overcome issues that caused by data accuracy, incompleteness and uncertainties. On the other hand, data uncertainty visualisation enhances reliable object detection. Fig. 1 represents different capability categories and their interrelationships for achieving automated and connected earthwork systems which are resilient against accidents. Advances and development of each capability class, and its associated technologies and approaches affect improvement of other capability classes.

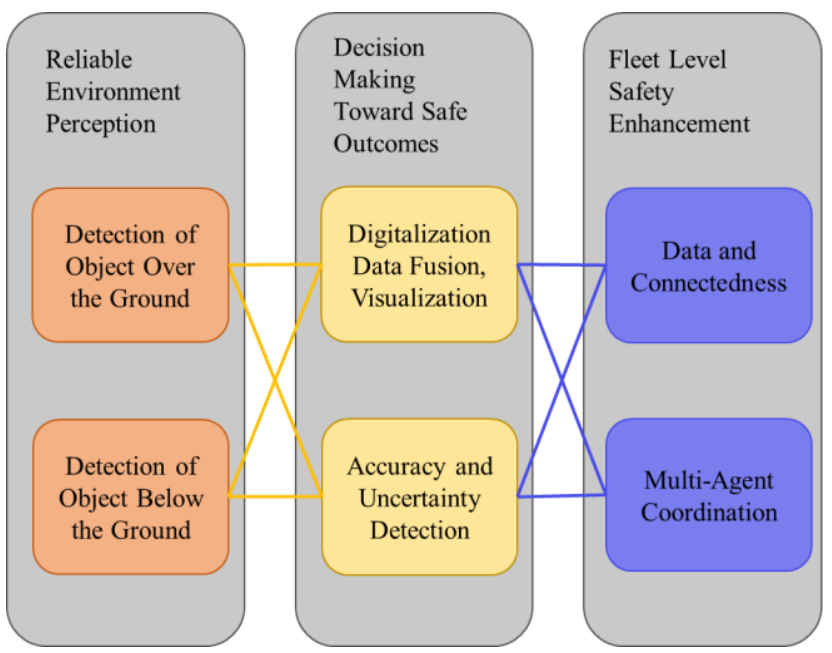

Figure 1 Required capabilities for accident-free automated and connected excavation systems.

\section{Levels of automation}

In general, automation systems should be described and activated in such a way that become a best-fit for the proficiency of both humans and machines. The term levels of automation (LOA) expresses human-machine interaction and cooperation (Vagia, Transeth and Fjerdingen, 2016). Each level determines a specific point in which a class of tasks is automated. This signifies that automation can alter across a sequence of intermediate levels at the two extremes, bounded by fully manual work, and a fully autonomous setting (Vagia, Transeth and Fjerdingen, 2016). Between these extremes, automation levels can be devised and a taxonomy can link previously proposed levels or add new ones (Vagia, Transeth and Fjerdingen, 2016). Furthermore, adaptive automation is needed and is analogous to dynamic function allocation, in which there is not a predetermined or fixed division of labour among human and machine agents, but it is context dependent, dynamic and flexible (Vagia, Transeth and Fjerdingen, 2016).

Terminology relating to "automation" and "autonomous" is critical especially as some publications use "autonomous" to mean "automation" (Shladover, 2017). "Autonomous" has the same meaning as "self-sufficiency" and "independence" and computer science literature has been using the "autonomous" word for systems that are capable of self-contained decision making (Shladover, 2017). While automation instructs system to act exactly in the way is programmed, without having any capability to act in any other way in different situations (Vagia, Transeth and Fjerdingen, 2016). However, when self-contained systems in the vehicle execute all functions, its automation system can accurately be considered as "autonomous". And if the vehicles acquire information via communications with other vehicles, or the infrastructure, they have "cooperative" automation systems (Shladover, 2017). The use cases identified in section IIIC can be related to different LOA. In the following four different LOA are proposed for earthwork technology roadmap, in which human intervention will be eliminated during transition from automated level toward autonomous level. Each LOA is a scenario that can take place based on the availability of technology innovations, and advancement in their accuracy levels.

\section{1) Automated earthwork}

In the basic level of automated earthmoving equipment such as an excavator is able to execute tasks automatically, and acquires various data automatically from different sensors and detects objects but each single action will be supervised via human intervention such as the operator of a machine. The earthmoving equipment has only environmental perception capability. 2D cabin mounted object visualization supports this stage of automation.

\section{2) Semi-autonomous earthwork}

At the semi-autonomous level, a remote operator utilises accurate and near-real time visualization to share operational control with the machine. The human can take control when managerial level decision making is required, such as in complex situations beyond the capability of the machine. The earthmoving equipment will have capabilities of: environmental perception and some degree of decision making toward safe outcomes. 


\section{3) Autonomous earthwork}

At the autonomous level the earthmoving equipment acts as a smart agent with precise environmental perception, and decision making capabilities to plan and control its own activities without the need for human intervention.

4) Connected and autonomous earthwork

At the connected, autonomous level the excavator has capabilities of environmental perception, decision making toward safety individually, and fleet level safety enhancement. This means it has connectedness ability and communication skill, to plan and control its activities within the context of other construction equipment and devices. At this level the excavator is able to receive and send information to various equipment on site, as well as to exchange information with any workers that enter into its safety zone. It can receive information from any utility network below the ground and plan its operational activities in real-time accordingly to avoid utility strikes.

Fig. 2 illustrates associations between use cases and different levels of automation. Currently, the use cases available to the basic level of "Automated earthwork" can be implemented into practice although there are some barriers in their effective implementation caused by data accuracy issues. Use cases may become redundant once a higher level of automation is achieved due to technology advancement. For example, visualization is no longer required for "Autonomous earthwork" since this level of automation does not require human intervention. Instead, reliable back up and classification of data is required in case the autonomous operation fails. Whilst Fig. 2 is clearly not a comprehensive illustration of all possible use cases, and indeed more granular levels of automation could be added, it illustrates the point that use cases are strongly correlated with levels of automation.

Now that the required capabilities and different levels of automation are defined, it is possible to develop a technology roadmap. To quantify the effect of automated and connected earthwork on system's safety and productivity, methods such as agent-based modelling can be used to represent various operation scenarios and use cases (Zhang and Hammad, 2011). In addition, the modelling of a roadmap as an agent-based model shows the development of capabilities like: reproducing history of technology, studying emerging properties, and coevolution of technologies and markets (Rinne, 2004). Agent based models can quantify metrics, self-organization of earthwork operations (Jabri and Zayed, 2017), and even boost construction firms' collaboration (Hammad et al., 2012) toward achieving automated and connected earthwork system at the earliest possible time. However, testbeds are vital to test, and approve the real and virtual validation of systems which add to automated and connected earthmoving equipment and environment functionalities.

\section{CONClusion}

The construction industry is going through significant industrial transformation, technology development and investment which are motivated by safety and productivity and enabled by automation and connectivity. This paper presents and discusses 1) industrial drivers, 2) specific capabilities, and
3) use cases, as components of an automated and connected earthwork technology roadmap which facilitate industrial transformation.

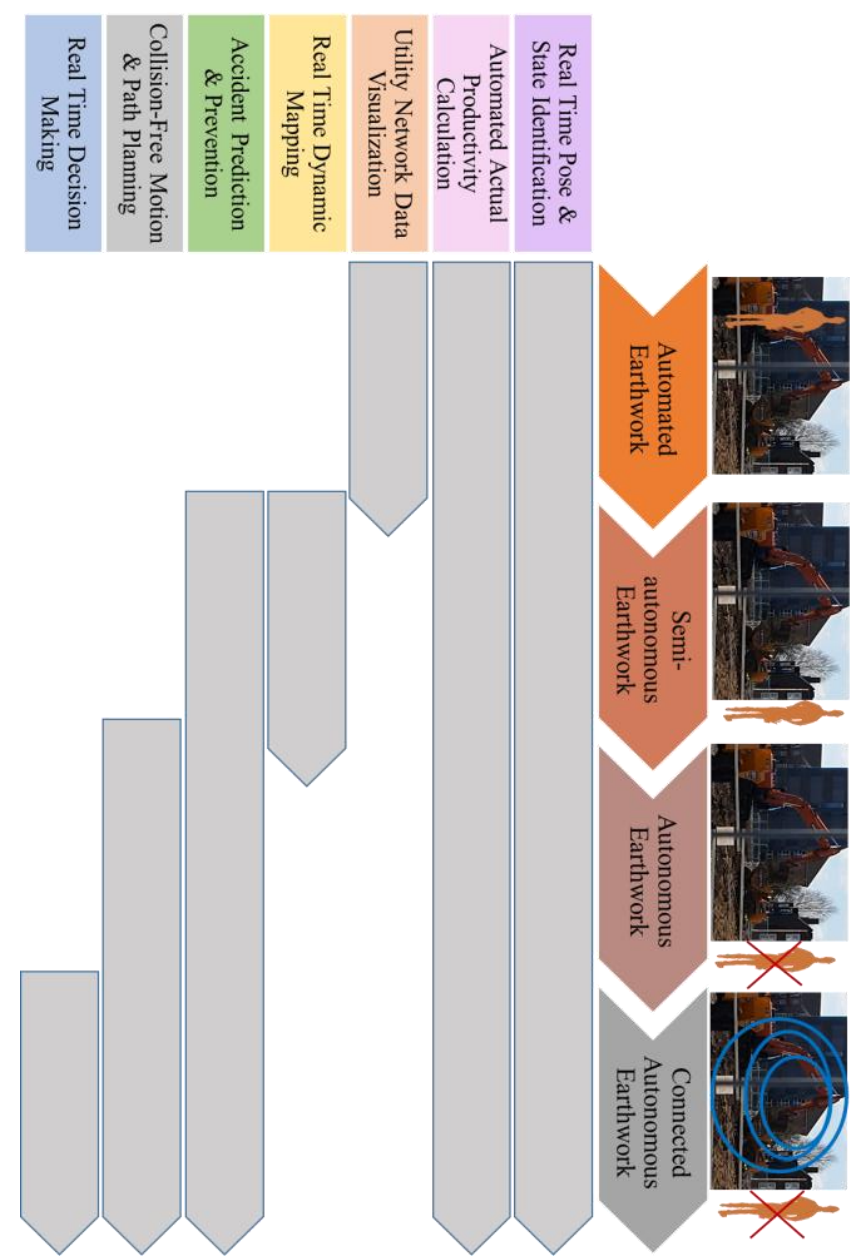

Figure 2 Automated and connected earthwork use cases, and their associated levels of automation.

This study combines the results of disintegrated and fragmented research in the area of automated and connected earthwork and categorises them under new capability levels.

Current technologies are providing the opportunity to implement use cases for the basic level of automation: "Automated earthwork". However, technologies with higher levels of accuracy are required for more advanced levels of automation. Improved data fusion algorithms, reliable and fast communication technologies, validated multi-agent coordination strategies can significantly affect the development and adaption of automated and connected earthwork systems.

This work can contribute to forming a single regulating platform for connected and automated earthwork by illustrating the required technology capabilities and use cases that can enhance safety and resilience of connected autonomous earthwork operation. This work also highlights technical requirements required of future technologies to achieve higher levels of autonomy and connectivity. In addition, this study has implications for the autonomy and connectivity technologies' market by providing a guide for the timing of investment in 
such technologies. To take this work forward, more use cases could be defined and the scope of technologies for purposes other than system safety and resilience could be included.

\section{REFERENCES}

Awolusi, I., Marks, E. and Hallowell, M. (2018) 'Wearable technology for personalized construction safety monitoring and trending: Review of applicable devices', Automation in Construction. Elsevier, 85(July 2016), pp. 96-106. doi: 10.1016/j.autcon.2017.10.010.

Azar, E. R. and Kamat, V. R. (2017) 'Earthmoving equipment automation: A review of technical advances and future outlook', Journal of Information Technology in Construction, 22, pp. 247-265. Available at: https://www.scopus.com/inward/record.uri?eid=2-s2.0-

85040561460\&partnerID=40\&md5=afbbf44a9db93a22bb11fe51e8bbd52c Behzadan, A. H., Dong, S. and Kamat, V. R. (2015) 'Augmented reality visualization: A review of civil infrastructure system applications', Advanced Engineering Informatics, 29(2), pp. 252-267. doi: 10.1016/j.aei.2015.03.005. Chae, S. and Yoshida, T. (2010) 'Application of RFID technology to prevention of collision accident with heavy equipment', Automation in Construction, 19(3), pp. 368-374. doi: 10.1016/j.autcon.2009.12.008. Choi, B., Hwang, S. and Lee, S. H. (2017) 'What drives construction workers' acceptance of wearable technologies in the workplace?: Indoor localization and wearable health devices for occupational safety and health', Automation in Construction, 84, pp. 31-41. doi: 10.1016/j.autcon.2017.08.005.

Dou, Q. et al. (2016) '3D buried utility location using a marching-crosssection algorithm for multi-sensor data fusion', Sensors (Switzerland), 16(11). doi: $10.3390 / \mathrm{s} 16111827$.

Fang, W. et al. (2018) 'Automated detection of workers and heavy equipment on construction sites: A convolutional neural network approach', Advanced Engineering Informatics, 37, pp. 139-149. doi: 10.1016/j.aei.2018.05.003. Gausemeier, J., Fink, A. and Schlake, O. (1998) 'Scenario Management: An Approach to Develop Future Potentials', Technological Forecasting and Social Change, 59(2), pp. 111-130. doi: 10.1016/S0040-1625(97)00166-2. Geum, Y. et al. (2015) 'Development of data-driven technology roadmap considering dependency: An ARM-based technology roadmapping', Technological Forecasting and Social Change, 91, pp. 264-279. doi: 10.1016/j.techfore.2014.03.003.

Gillespie, G. (2017) 'Intelligent Connected Vehicle Roadmap', (September). Hammad, A. et al. (2012) 'Towards the smart construction site: Improving productivity and safety of construction projects using multi-agent systems, real-time simulation and automated machine control', Proceedings - Winter Simulation Conference. IEEE, pp. 1-12. doi: 10.1109/WSC.2012.6465160. Hansen, C. et al. (2016) 'The future of rail automation: A scenario-based technology roadmap for the rail automation market', Technological Forecasting and Social Change, 110This pa, pp. 196-212. doi: 10.1016/j.techfore.2015.12.017.

Highways England, TRL, I. (2020) 'Connected and Autonomous Plant (CAP) Roadmap to 2035', \{Highways England\}. Available at: https://assets.highwaysengland.co.uk/Innovation+Hub/CAP+roadmap.pdf. Hollnagel, E. (2014) 'Resilience engineering and the built environment', Building Research and Information. Taylor \& Francis, 42(2), pp. 221-228. doi: 10.1080/09613218.2014.862607.

Hosseini, S., Barker, K. and Ramirez-Marquez, J. E. (2016) 'A review of definitions and measures of system resilience', Reliability Engineering and System Safety, 145, pp. 47-61. doi: 10.1016/j.ress.2015.08.006.

Huang, Y. et al. (2018) 'A technology delivery system for characterizing the supply side of technology emergence: Illustrated for Big Data \& Analytics', Technological Forecasting and Social Change, 130, pp. 165-176. doi: 10.1016/j.techfore.2017.09.012.

Huber, S. A. et al. (2007) 'Resilience Engineering : New directions for measuring and maintaining safety in complex systems 4 th Progress Report, November 2007 Resilience Engineering : New directions for measuring and maintaining safety in complex systems Fourth Progress Report', (November). Hussain, M., Tapinos, E. and Knight, L. (2017) 'Scenario-driven roadmapping for technology foresight', Technological Forecasting and Social Change, 124, pp. 160-177. doi: 10.1016/j.techfore.2017.05.005.

i3P, Highways England, TRL, C. (2020) 'Delivering digital infrastructure, infrastructure client leadership to deliver digital transformation of construction processes.', \{Highways England\}. Available at: https://s3.eu-west2.amazonaws.com/assets.highwaysengland.co.uk/Innovation+Hub/Delivering +digital+infrastructure+-+making+it+happen.pdf.

Jabri, A. and Zayed, T. (2017) 'Agent-based modeling and simulation of earthmoving operations', Automation in Construction. Elsevier, 81(May), pp.
210-223. doi: 10.1016/j.autcon.2017.06.017.

Jirgl, M., Bradac, Z. and Fiedler, P. (2019) 'Adaptive Human Control Model and its Usability in Modeling of Human-in-the-loop Cyber Physical Systems', IFAC-PapersOnLine. Elsevier Ltd, 52(27), pp. 415-420. doi: 10.1016/j.ifacol.2019.12.697.

Kim, C. et al. (2009) 'Developing a technology roadmap for construction R\&D through interdisciplinary research efforts', Automation in Construction, 18(3), pp. 330-337. doi: 10.1016/j.autcon.2008.09.008.

Kim, H. et al. (2018) 'Detecting Construction Equipment Using a RegionBased Fully Convolutional Network and Transfer Learning', Journal of Computing in Civil Engineering, 32(2). doi: 10.1061/(ASCE)CP.19435487.0000731 .

Kim, J. et al. (2018) 'Modular data communication methods for a robotic excavator', Automation in Construction, 90, pp. 166-177. doi: 10.1016/j.autcon.2018.02.007.

Kolera, B. T. and Bernold, L. E. (2006) 'Intelligent Utility Locating Tool for Excavators.', Journal of Construction Engineering \& Management. American Society of Civil Engineers, 132(9), pp. 919-927. Available at: http://10.0.4.37/(ASCE)0733-9364(2006)132:9(919).

Lee, C., Song, B. and Park, Y. (2015) 'An instrument for scenario-based technology roadmapping: How to assess the impacts of future changes on organisational plans', Technological Forecasting and Social Change. Elsevier Inc., 90(PA), pp. 285-301. doi: 10.1016/j.techfore.2013.12.020.

Lee, H. and Geum, Y. (2017) 'Development of the scenario-based technology roadmap considering layer heterogeneity: An approach using CIA and AHP', Technological Forecasting and Social Change, 117, pp. 12-24. doi: 10.1016/j.techfore.2017.01.016.

Lee, J. H., Phaal, R. and Lee, S. H. (2013) 'An integrated service-devicetechnology roadmap for smart city development', Technological Forecasting and Social Change, 80(2), pp. 286-306. doi: 10.1016/j.techfore.2012.09.020. Macchi, L., Hollnagel, E. and Leonhard, J. (2009) 'Resilience Engineering approach to safety assessment: an application of FRAM for the MSAW system', EUROCONTROL Safety R\&D Seminar, p. 12.

Olhoeft, G. R. (2000) 'Maximizing the information return from ground penetrating radar', JOURNAL OF APPLIED GEOPHYSICS, 43(2-4), pp. 175-187. doi: 10.1016/S0926-9851(99)00057-9.

Phaal, R. (2004) 'Technology roadmapping - A planning framework for evolution and revolution', Technological Forecasting and Social Change, 71(1-2), pp. 5-26. doi: 10.1016/S0040-1625(03)00072-6.

Rinne, M. (2004) 'Technology roadmaps: Infrastructure for innovation', Technological Forecasting and Social Change, 71(1-2), pp. 67-80. doi: 10.1016/j.techfore.2003.10.002.

Shirali, G. H. A. et al. (2012) 'Assessing resilience engineering based on safety culture and managerial factors', Process Safety Progress, 31(1), pp. 1718. doi: $10.1002 /$ prs. 10485 .

Shladover, S. E. (2017) 'Connected and automated vehicle systems: Introduction and overview', Journal of Intelligent Transportation Systems, pp. 1-11. doi: 10.1080/15472450.2017.1336053.

Soltani, M. M., Zhu, Z. and Hammad, A. (2017) 'Skeleton estimation of excavator by detecting its parts', Automation in Construction. Elsevier, 82(July), pp. 1-15. doi: 10.1016/j.autcon.2017.06.023.

Soltani, M. M., Zhu, Z. and Hammad, A. (2018) 'Framework for Location Data Fusion and Pose Estimation of Excavators Using Stereo Vision', Journal of Computing in Civil Engineering, 32(6). doi: 10.1061/(ASCE)CP.19435487.0000783.

$\mathrm{Su}$, X. et al. (2013) 'Uncertainty-aware visualization and proximity monitoring in urban excavation: a geospatial augmented reality approach', Visualization in Engineering, 1(1). doi: 10.1186/2213-7459-1-2. Tajeen, H. and Zhu, Z. (2014) 'Image dataset development for measuring construction equipment recognition performance', Automation in Construction, 48, pp. 1-10. doi: 10.1016/j.autcon.2014.07.006.

Talmaki, S. and Kamat, V. R. (2014) 'Real-time hybrid virtuality for prevention of excavation related utility strikes', Journal of Computing in Civil Engineering, 28(3). doi: 10.1061/(ASCE)CP.1943-5487.0000269.

Vagia, M., Transeth, A. A. and Fjerdingen, S. A. (2016) 'A literature review on the levels of automation during the years. What are the different taxonomies that have been proposed?', Applied Ergonomics, pp. 190-202. doi: 10.1016/j.apergo.2015.09.013.

Vähä, P. et al. (2013) 'Extending automation of building construction Survey on potential sensor technologies and robotic applications', Automation in Construction, pp. 168-178. doi: 10.1016/j.autcon.2013.08.002.

Vahdatikhaki, F. et al. (2017) 'Enhancing coordination and safety of earthwork equipment operations using Multi-Agent System', Automation in Construction, 81, pp. 267-285. doi: 10.1016/j.autcon.2017.04.008. Vahdatikhaki, F., Hammad, A. and Siddiqui, H. (2015) 'Optimization-based 
excavator pose estimation using real-time location systems', Automation in Construction, 56, pp. 76-92. doi: 10.1016/j.autcon.2015.03.006.

Viljamaa, E. and Peltomaa, I. (2014) 'Intensified construction process control using information integration', Automation in Construction. Elsevier B.V., 39, pp. 126-133. doi: 10.1016/j.autcon.2013.08.015.

Zekavat, P. R., Moon, S. and Bernold, L. E. (2014) 'Securing a wireless site network to create a BIM-allied work-front', International Journal of

Advanced Robotic Systems, 11(1), pp. 1-15. doi: 10.5772/58441.

Zhang, C. and Hammad, A. (2011) 'Multiagent Approach for Real-Time Collision Avoidance and Path Replanning for Cranes', Journal of Computing in Civil Engineering, 26(6), pp. 782-794. doi: 10.1061/(asce)cp.19435487.0000181 .

Zhang, L. et al. (2017) 'Perceiving safety risk of buildings adjacent to tunneling excavation: An information fusion approach', AUTOMATION IN CONSTRUCTION, 73, pp. 88-101. doi: 10.1016/j.autcon.2016.09.003.

Zhang, Y. et al. (2016) 'Technology roadmapping for competitive technical intelligence', Technological Forecasting and Social Change, 110, pp. 175186. doi: 10.1016/j.techfore.2015.11.029. 